\title{
Die Situmetrie (Einregelungsmessung) als morphologische Untersuchungsmethode
}

\author{
Gerhard Furrer und Fritz Bachmann
}

\section{Das Wesen der Situmetrie}

Jeder Massenbewegung wohnen eigene Gesetzmäßigkeiten inne, und dem dislozierten Material prägen sich die Eigentümlichkeiten der jeweiligen Transportart auf. Darum bleibt die Sedimentation nicht dem Zufall überlassen, sondern erfolgt nach bestimmten, dem jeweiligen Transportmechanismus adäquaten Ordnungsprinzipien. Allerdings sprechen nicht alle der lockeren Materialkomponenten in gleicher Weise auf die wirksamen Kräfte an. Infolge ihrer zu bescheidenen Dimensionen erweisen sich die kleineren Bestandteile als inkompetent. Zu sehr lassen sie sich auch von Nebenwirkungen beeinflussen, die nicht unmittelbar mit der eigentlichen Bewegung zusammenhängen. So unterliegen sie beispielsweise der Stauwirkung von voluminöseren Komponeten oder der "Turbulenz» beim Umfließen von Hindernissen. Sie fallen darum als «Füllmaterial» bei den situmetrischen Untersuchungen auBer Betracht. Verschieden verhalten sich aber auch die größeren Bestandteile, die der Dislokation beträchtlichen Widerstand entgegenzusetzen vermögen. Darum beschränkt sich die Untersuchung im allgemeinen auf steinige Partikel von 2 bis $20 \mathrm{~cm}$ größtem Durchmesser (Poser, 1951). Doch dürfen nur Steine berücksichtigt werden, die eine deutlich erkennbare Längsachse aufweisen, denn nur solche werden durch die erzeugten Drehmomente in eine durch exakte Messung faßbare räumliche Anordnung gezwungen. Somit offenbart sich das Ordnungsprinzip, dem die Sedimentation gehorcht, in der Lage, welche die Längsachsen solcher gestreckter Gerölle einnehmen. A us der Bewegung resultiert die Einregelung, d. h. die einer bestimmten Transportart gemäß sich einstellende Regel- oder gar Gesetzmäßigkeit in der Orientierung der Steinlängsachsen.

Als Situmetrie (Bachmann, 1966) bezeichnen wir die Methode, welche die quantitative Erfassung der Einregelung ermöglicht und dann durch Auswertung der Ergebnisse fundierte Aussagen erlaubt. Die Bedeutung der Situmetrie beruhte bisher im wesentlichen darauf, daß sie entweder zur Bestimmung der Bewegungsrichtung eines an sich bekannten sedimentierenden Transportmediums diente oder aber bei bekannter Transportrichtung zur Ermittlung der Verfrachtungsart. Wir erweitern diesen beschränkten Anwendungsbereich dahin, daß wir die
Einregelung als ein charakteristisches inneres Strukturmerkmal bestimmter morphologischer Formen auffassen (Bachmann, 1966). Eingehende situmetrische Untersuchungen, z. B. an rezenten Strukturbodenformen (Furrer, 1965 und Elsaßer, 1966), führen zur Erkenntnis, daß diesen Formen gewisse situmetrische Regelmäßigkeiten eigen sind, die als entscheidende Indizien gewertet werden dürfen. Die Bearbeitung rezenter Formen aber bleibt nicht Selbstzweck, denn sie liefert in hohem Maße die Grundlagen, auf denen die Untersuchung der entsprechenden fossilen Formen basiert. Fossile Formen präsentieren sich in der Regel unter wesentlich ungünstigeren Beobachtungsverhältnissen. Meist sind sie unter jüngeren Ablagerungen verborgen und deshalb nur in Profilschnitten ersichtlich. Um so größere Bedeutung kommt deshalb den inneren Strukturmerkmalen zu, die eine sichere Diagnose von aufgeschlossenen Formen erlauben. In dieser Beziehung erhält die Einregelung unter den in Betracht kommenden Merkmalen das Primat, und so wird die Situmetrie zu einer fundamentalen morphologischen Untersuchungsmethode.

\section{Die Entwicklung der Methode}

Die Situmetrie ist eine Feldmethode. Unmittelbar am Objekte selbst erfolgt dessen Untersuchung. Es handelt sich darum, bei jeder Messung die Orientierung von $100 \mathrm{Geröllelängsachsen} \mathrm{zu} \mathrm{ermitteln.} \mathrm{Diese}$ Zahl wird nicht nur gewählt, weil sie ohne Umrechnung eine prozentuale Aufgliederung in Gruppen erlaubt, sondern auch, um das Ergebnis hinreichend repräsentativ zu gestalten. Versuche am Beispiel von Solifluktionsformen haben ergeben, daß bei 100 beobachteten Längsachsen bei über $80 \%$ aller untersuchten Formen der Solifluktionscharakter zu erkennen war. Die Orientierung der Geröllelängsachsen läßt sich in einer Ebene durch ein Winkelfeld von $180^{\circ}$ erfassen, das dann entsprechend unterteilt werden kann. Diese Unterteilung hat im Laufe der Zeit verschiedene Veränderungen erfahren.

Die für die anfängliche Entwicklung der Situmetrie maßgebenden Autoren sind Richter (1932) sowie Poser und Hövermann (1951). Richter unternahm die situmetrische Untersuchung mit dem Kompaß und bezog die Orientierung der Geröllelängsachsen auf die Nordrichtung, indem er das Azimut be- 
stimmte. Hierauf faßte er die Einzelmessungen in Winkelsektoren von $10^{\circ}$ Öffnung zusammen, also die Azimute von $0^{\circ}$ bis $10^{\circ}$, von $10^{\circ}$ bis $20^{\circ}$, usw. Poser und Hövermann vereinfachten die Methode durch Anwendung einer halbkreisförmigen Meßtafel, die von der Mittellinie aus in Sektoren von $30^{\circ}$ eingeteilt war. Die Einzelbeobachtungen werden in 3 Richtungsgruppen zusammengefaßt:

Gruppe I beidseits bis zu $30^{\circ}$ von der Mittellinie abweichend.

Gruppe II beidseits $30^{\circ}$ bis $60^{\circ}$ von der Mittellinie abweichend.

Gruppe III beidseits $60^{\circ}$ bis $90^{\circ}$ von der Mittellinie abweichend.

Dazu kommt noch die Regelungsgruppe IV, diejenigen Gerölle umfassend, deren Längsachsen steilschräg in die Tiefe weisen, wobei Körber (1956) den an sich willkürlichen Grenzwert von $40^{\circ} \mathrm{Nei}-$ gung zur Horizontalebene festzulegen versuchte, während wir eine Neigung von $45^{\circ}$ zur Tafelebene fixiert haben.

Solange die Situmetrie nur zur Bestimmung von Transportmedien oder Transporteinrichtungen eingesetzt wurde, mochte die sehr einfache und darum in hohem Maße feldtaugliche Unterteilung von Poser und Hövermann durchaus genügen. Zur Ermittlung von inneren Strukturmerkmalen bestimmter morphologischer Formen hingegen drängte sich eine feinere Gliederung auf. Unter den beiden Verfassern sah sich zunächst Furrer (1965) veranlaßt, für die situmetrische Untersuchung von rezenten Solifluktionsformen auf der oben beschriebenen Meßtafel die Sektoren durch Winkelteilung zu halbieren, weil nur so die subtileren Formmerkmale zum Ausdruck kommen konnten. Von der Mittelachse der Meßtafel ausgehend, wurden somit links und rechts je die Sektoren I alpha und I beta, II alpha und II beta sowie III alpha und III beta erlangt. Bachmann (1966) hat hierauf bei der Bearbeitung fossiler Strukturböden die $15^{\circ}$-Sektoren, links oben beginnend, fortlaufend numeriert und damit auf der Meßtafel deren zwölf erhalten, wozu noch, der oben erwähnten Gruppe IV entsprechend, ein Bereich Nummer 13 trat. Für zusammenfassende Übersichten, vergleichende Betrachtungen und insbesondere für die Darstellung der Meßergebnisse in Dreieckkoordinaten aber griffen wir stets wieder auf die Gruppen I, II, III und IV nach Poser und Hövermann zurück. Obwohl unsere neue Unterteilung zunächst eine befriedigende Erfassung der später zu erläuternden situmetrischen Strukturmerkmale zu gewährleisten schien, erwies es sich doch als unumgänglich, das Einteilungsprinzip weiter zu vervollkommnen. Anlaß dazu gab die Sammelgruppe IV, bzw. der Einregelungsbereich 13, werden doch die hierein fallenden Komponenten nur in bezug auf die Neigung ihrer Längsachsen zur Tafelebene eingemessen, jedoch nicht in bezug auf die effektive Orientierung ihrer Längsachsen, die trotz der Steilstellung besteht. Besonders dort, wo sehr viele Einzelgerölle in diesen Bereich fallen, möglicherweise sogar mehr als 50\%, wie dies beispielsweise bei den hier nicht behandelten Füllungen von Eiskeilspal-

\section{A}
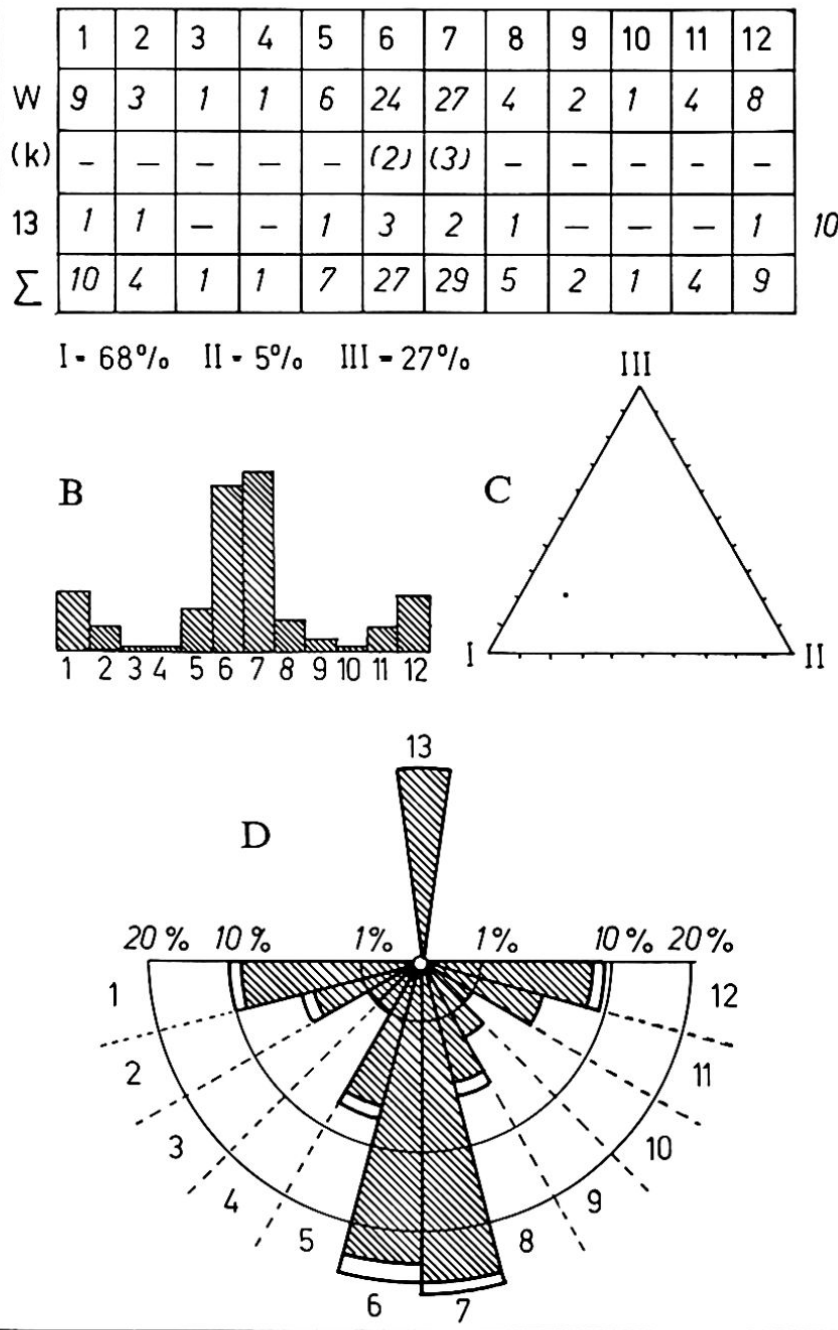

Figur 1. Verschiedene Darstellungsmethoden fü situmetrische Ergebnisse. A: Tabellarisches Meß protokoll, B: Säulendiagramm, C: Dreieckkoordina ten, D: Situgramm. Das Beispiel entstammt einen fossilen Steinstreifen im Ruckfeld AG

ten der Fall sein kann, drängt sich die Aufteilun: des Bereiches 13 auf die 12 Sektoren der Meßtafe auf. Nur so können schließlich die situmetrischeı Charakteristika von Formen voll zum Ausdrucl kommen, und nur auf diese Weise wird die untes erläuterte Darstellung der Meßergebnisse in Drei eckkoordinaten einwandfrei.

So werden also nunmehr die steilstehenden Längs achsen auf die Tafelebene projiziert, d. h., es wirs die Orientierung ermittelt, die sie einnähmen, wenı sie in der Tafelebene lägen. Allerdings eliminiereı wir die Tatsache der Steilstellung nicht, denn ein Häufung von so zur Tafelebene liegenden Längs achsen kann ebenso sehr formales Charakteristikun sein wie die orientierungsmäßige Verteilung in de Tafelebene selbst. Darum werden die steilstehende Gerölle, die in den Bereich 13 fallen, gemäß den situmetrischen Meßprotokoll (Fig. 1) so notiert, dal die Ermittlung des prozentualen Anteils der Sam melgruppe IV bzw. 13 jederzeit möglich ist. Senk 
recht im Boden steckende Komponenten allerdings sind auf diese Weise nicht mehr darstellbar. Doch hat die bisherige Erfahrung gezeigt, daß eine derartige Stellung so selten vorkommt, daß die betreffenden Gerölle analog denen, die keine ausgeprägte Längsachse aufweisen, unberücksichtigt bleiben dürfen.

Gleichzeitig können gemäß Figur 1 bei der Messung auch Angaben über die Kantenstellung plattiger Steine festgehalten werden. Vorläufige Meßergebnisse an Solifluktionsformen lassen diesbezüglich erkennen, daß in an sich plattigen Komponenten, z. B. in Bündner Schiefer und Flysch, die Kantenstellung teilweise recht prägnant in Erscheinung treten kann, ohne aber das Gesamtergebnis zu beeinträchtigen (mdl. Mitt. H. Elsasser).

\section{Die Darstellung der Meßergebnisse}

Die bei der Feldarbeit gewonnenen Meßergebnisse bedürfen zur weiteren Verarbeitung einer entsprechenden graphischen Darstellung. Bisher war es üblich, die prozentualen Anteile der Sammelgruppen I bis IV wie Richter als nebeneinandergestellte Säulen von zu den ermittelten Werten proportionaler Höhe zu veranschaulichen. Damit gelangen wohl die numerischen Werte zur Darstellung. Die Orientierung jedoch, die ja durch die Messung in erster Linie erfaßt werden soll, kommt aber so nicht zum Ausdruck. Das Säulendiagramm wird somit dem ermittelten Ergebnis nur halbwegs gerecht. Darum mußte nach besseren Darstellungsmöglichkeiten gesucht werden. An Versuchen, prozentuale Anteile und Richtung gleichzeitig graphisch zu erfassen, fehlt es nicht. Wir verweisen auf die Arbeiten von Rydquist (1960) und Fischer (1966).

\section{a) Das Situgramm}

Die von uns schließlich zur Veranschaulichung situmetrischer Befunde mit Erfolg angewandte Darstellungsform, nämlich das Situgramm, geht auf Bachmann (1966) zurück. Grundlage ist eine verkleinerte Wiedergabe der Meßtafel mit ihren zwölf Sektoren. Die prozentualen Anteile der einzelnen Einregelungsbereiche sind durch Sektoren, deren Flächen proportional zu den jeweiligen numerischen Werten gehalten sind, veranschaulicht. So erfolgt die Verbindung der auf die Tafelebene bezogenen Orientierung mit der jeweiligen Anzahl der Komponenten. Das Situgramm gibt also die Einregelung unmittelbar wieder. Von großem Vorteil ist zudem, $\mathrm{da} ß$ derartige Situgramme genau orientiert in Situationspläne eingezeichnet werden können. Die steilgestellten Gerölle im Einregelungsbereich IV bzw. 13 werden als senkrecht nach oben gerichteter Sektor von ebenfalls $15^{\circ}$ Öffnung einbezogen. Die Projektion dieser Gerölle auf die Tafelebene gelangt gemäß Figur 1 zur Darstellung, indem deren Anteile durch nicht flächengetönte Ringsektoren den ursprünglichen Sektoren angefügt werden.

\section{b) Dreieckkoordinaten}

Am ausgeprägtesten kommen die situmetrischen Regelmäßigkeiten bestimmter morphologischer Formen zum Ausdruck, wenn die Meßergebnisse in Dreieckkoordinaten zur Darstellung gelangen (Bachmann, 1966). Allerdings lassen sich auf diese Weise nur drei der Sammelgruppen I bis IV verarbeiten. Zwangsläufig muß darum einer der Werte eliminiert werden. Bei der Untersuchung von Strukturböden, Solifluktionsschutt und Flußschottern, auf welche hier allein verwiesen werden soll, ergab sich, daß die Sammelgruppe IV nur eine untergeordnete Rolle spielt. Meist liegen nur wenig Gerölle deutlich gegen die Tafelebene geneigt, und deren Zahl erhält keinen primären Aussagewert. Deshalb wurde bei früheren Messungen, die noch nicht nach der weiterentwickelten Methode vorgenommen werden konnten, der Anteil der steilstehenden Komponenten proportional auf die Sammelgruppen I, II und III aufgeteilt (Bachmann, 1966). Nach der oben beschriebenen verbesserten Meßmethode jedoch fällt diese Umrechnung dahin. Die steilgestellten Partikel werden ja nun durch die Vertikalprojektion auf die Meßtafelebene ihrer effektiven Orientierung gemäß auf die Sektoren 1 bis 12 verteilt.

Ein Beispiel möge darlegen, wie relativ geringfügig sich die Werte unterscheiden, wenn an gleicher Stelle die Messung sowohl nach der ursprünglichen als auch nach der verbesserten Methode erfolgt. Es handelt sich dabei um eine situmetrische Messung in einem fossilen Steinstreifen, bei der sich ein relativ hoher Wert in der Gruppe IV einstellte:

Aufnahme nach ursprünglicher Methode

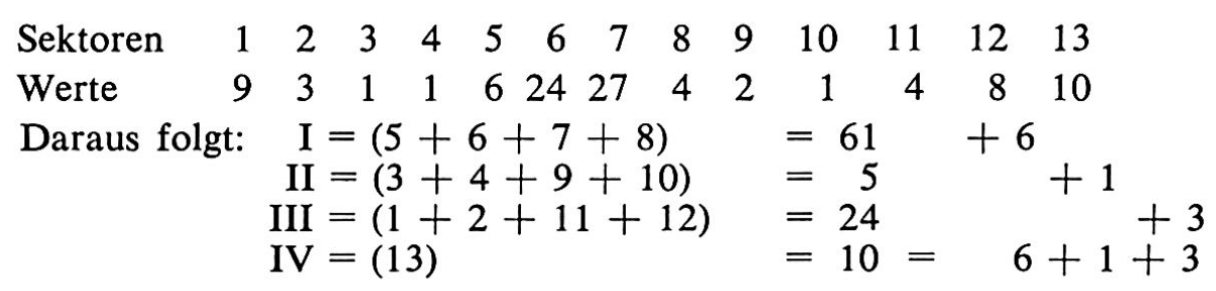

Werte für Dreieckkoordinaten: $\mathrm{I}=67 \quad$ II $=6 \quad$ III $=27$ 
Aufnahme nach verbesserter Methode

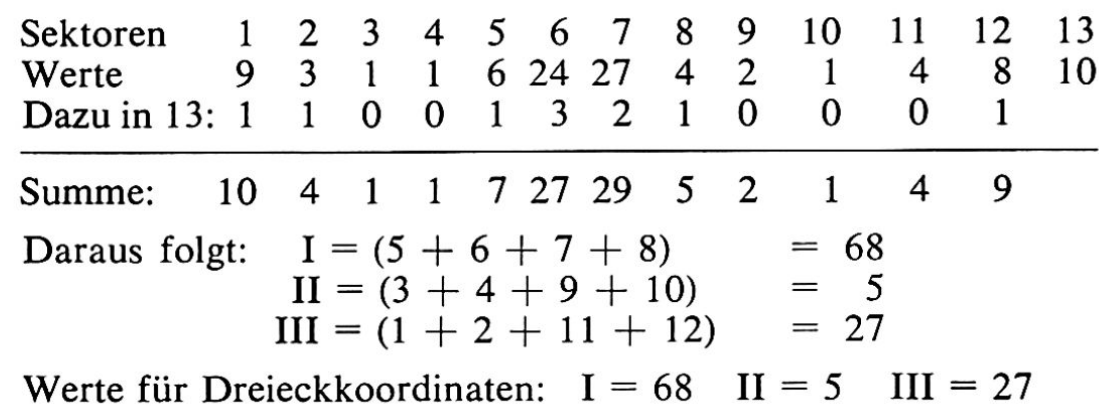

Somit dürfen, mindestens solange der Wert der Sammelgruppe IV im Vergleich zu den übrigen Werten gering bleibt, die nach der ursprünglichen Methode ermittelten Meßergebnisse unbeschadet auf die angedeutete Weise umgerechnet werden. Es zeigt sich, daß die steilgestellten Komponenten nach den gleichen Einregelungsprinzipien angeordnet liegen wie die in die Meßtafelebene eingespielten. Bei vergleichenden Betrachtungen lassen sich somit Ergebnisse, die noch nach der alten Methode gewonnen wurden, ohne Schwierigkeiten mit solchen, denen die neue Methode zu Grunde liegt, in Beziehung setzen.

Bei der Darstellung der Meßergebnisse in Dreieckkoordinaten liefert jede Messung einen Punkt. Wenn sich in der Einregelung einzelner Formen und Ablagerungen gewisse Regelmäßigkeiten ausprägen, beispielsweise daß bei Steinstreifen die Werte in der Sammelgruppe I stets größer sind als in der Sammelgruppe III und diese wiederum stets die Werte der Sammelgruppe II übersteigen, scharen sich die Bildpunkte in einer bestimmten Partie der Dreiecksfläche. Es zeichnen sich also, den situmetrischen Eigentümlichkeiten gemäß, einzelne Felder ab, die sich als bestimmten Formen und Ablagerungen zugeordnet erweisen. Solange eine bestimmte Regelmäßigkeit erfüllt bleibt, fallen infolgedessen alle Bildpunkte innerhalb die Begrenzung eines solchen Feldes.

$$
\text { (2) } \quad \mathrm{X}^{2} .95=\frac{\left(33^{1 / 3}-\mathrm{a}_{1}\right)^{2}+\left(33^{1 / 3}-\mathrm{a}_{2}\right)^{2}+33^{1 / 3}-\left[100-\left(\mathrm{a}_{1}+\mathrm{a}_{2}\right)\right]^{2}}{33^{1 / 3}}=6,0
$$

6,0 ist der Tafelwert für $\mathrm{X}^{2} .95$ bei 2 Freiheitsgraden.

Aufgelöst nach $a_{1}$ ergeben sich $a_{2}$-Werte zwischen 21,85 und 44,82, und damit lassen sich die Koordinaten von 4 Punkten ermitteln, in denen zu den Koordinatenachsen parallel verlaufende Tangenten die Begrenzung des "Vertrauensbereiches" der Gleichverteilung berühren, nämlich

$$
\begin{array}{lll}
P_{1}(27,59 / 44,82) & P_{3}(44,82 / 27,59) \\
P_{2} & (39,07 / 21,85) & P_{4}(21,85 / 39,07)
\end{array}
$$

Bei Ergebnissen, deren punktförmige Abbildung mit Hilfe von Dreieckkoordinaten außerhalb des so gewonnenen Kreises zu liegen kommen, ist mit einer Wahrscheinlichkeit von 95 Prozent Gleichverteilung auszuschließen.

Wie bereits erwähnt, ordnen sich die situmetrische
Sind derartige Regelmäßigkeiten durch eine große Zahl von Messungen offenbar geworden, so können sie als Basis zur Beurteilung von neuen Messungen dienen. Mit Hilfe des $X^{2}$-Testes lassen sich Abweichungen von einer theoretischen oder angenommenen Verteilung, z. B. von der als situmetrische Regelmäßigkeit erkannten, mathematisch fassen. Ebenso lassen sich auf gleicher Grundlage die für: eine bestimmte Verteilung geltenden "Vertrauensbereiche» berechnen.

Als Beispiel soll der "Vertrauensbereich" einer gleichmäßigen Verteilung (Gleichverteilung) für $\mathrm{X}^{2} .95$ ermittelt und in Dreieckkoordinaten dargestellt werden:

Es seien $h_{1}, h_{2}$ und $h_{3}$ die theoretischen Häufigkeiten in den Sammelgruppen I, II und III, $a_{1}, a_{2}$ und $a_{3}$ die beobachteten. Es gilt nun die Gleichung

(1) $\mathrm{X}^{2}=\frac{\left(\mathrm{h}_{1}-\mathrm{a}_{1}\right)^{2}}{\mathrm{~h}_{1}}+\frac{\left(\mathrm{h}_{2}-\mathrm{a}_{2}\right)^{2}}{\mathrm{~h}_{2}}+\frac{\left(\mathrm{h}_{3}-\mathrm{a}_{3}\right)^{2}}{\mathrm{~h}_{3}}$

Deren graphisches Bild ergibt im Dreieckkoordina. tensystem eine Ellipse, in Ausnahmefällen, wie z. B. bei Gleichverteilung, einen Kreis.

Da eine situmetrische Messung auf 100 Einzelbe. obachtungen basiert, ergibt $\mathrm{a}_{1}+\mathrm{a}_{2}+\mathrm{a}_{3}=100$ und für Gleichverteilung ist $h_{1}=h_{2}=h_{3}=331 /$ : zu setzen.

In Gleichung (1) eingesetzt, ergibt sich folgende Ausdruck:

Messungen repräsentierenden Punkte verschiede. nen Partien der Dreiecksfläche ein, je nachdem, ot Solifluktionsformen oder aber Flußschotter einge messen worden sind. Es zeichnet sich also ein Felc der Solifluktionsformen und ein solches der Fluß schotter ab. Der oben als Beispiel berechnete «Ver trauensbereich» erlaubt es nun, bei der Feldarbei auf einfache Weise und mit hoher Wahrscheinlich keit eine Gleichverteilung auszuschließen. Liegt so dann der einer Messung entsprechende Punkt au ßerhalb des Gleichverteilungsfeldes, läßt sich in der meisten Fällen anhand seiner Lage im Dreieck ein Diagnose stellen. Wie dies möglich ist, soll im fol genden anhand der uns durch eine große Zah von Messungen bereits sehr vertrauten Solifluktions formen und an Flußschottern dargelegt werden. 
Die Methode, die nun in ihren wesentlichen Zügen dargelegt worden ist, kann bei der Feldarbeit fundamentale Bedeutung erlangen. Nicht alle der Formen und Ablagerungen, mit denen sich die Geomorphologie zu befassen hat, sind ohne weiteres erkennbar. Beispielsweise haben sie noch keinen optimalen Entwicklungsgrad erreicht, weshalb die optische Ausprägung mangelhaft erscheint. Möglicherweise kann auch das Material, aus dem die Formen aufgebaut sind, Modifikationen bewirken, die vom idealen Erscheinungsbild abweichen. Auch zeigt die Erfahrung, daß Strukturböden oder Solifluktionsformen etwa infolge ihrer geringen Dimensionen oder fehlender Farbunterschiede zwischen Steinrahmen und Feinmaterial, wie z. B. in Serpentingebieten oder auch auf Schotterflächen, übersehen werden. Schließlich aber gelten diese Schwierigkeiten vor allem für fossile Formen, die sich sozusagen immer der unmittelbaren Beobachtung entziehen und in den meisten Fällen bei Aufschlüssen nur im Schnitt sichtbar werden. Trotz dieser und ähnlicher Unzulänglichkeiten ist doch in der Regel auf Grund eingehender situmetrischer Untersuchungen eine Entscheidung möglich, denn wenn gewisse Regelmäßigkeiten sich einstellen, darf mit hoher Wahrscheinlichkeit eine Form oder eine Ablagerung als solche erkannt und entsprechend angesprochen werden.

\section{Solifluktionsformen}

a) Die situmetrischen Eigentümlichkeiten von Steinstreifen

Úber rezente und fossile Steinstreifen liegen bisher die meisten Meßergebnisse vor, und an diesen Formen haben sich die situmetrischen Eigentümlichkeiten sehr prägnant offenbart (Furrer, 1965 und 1966; Bachmann, 1966; Elsasser, 1966). Am Beispiel der Steinstreifen ist auch klar geworden, daß die fossilen Formen den gleichen situmetrischen Prinzipien unterworfen sind wie die rezenten, d. h., daß die an rezenten Formen gewonnenen Erkenntnisse auf die Untersuchung der fossilen Formen übertragen werden dürfen. Diese Tatsache erlaubt uns heute, fossile Steinstreifen als solche zu erkennen und zu lokalisieren und außerdem fossile Strukturbodenformen mit hinreichender Sicherheit von Schottern zu unterscheiden.

Selbstverständlich ist eine gewisse Anzahl von Messungen erforderlich, bis eine Regel sich herausschälen läßt. Die Erfahrung hat gezeigt, daß Mittelwerte, gewonnen aus $30 \mathrm{Meßergebnissen,} \mathrm{basierend} \mathrm{auf}$ je 100 Einzelbeobachtungen, im allgemeinen ein mehr oder weniger symmetrisches Situgramm er- geben, d.h., daß die jeweils sich entsprechenden Sektoren auf der linken und der rechten Seite der Mittellinie (Radius zwischen 6 und 7) weitgehend gleiche Belastungen erhalten (vgl. Sammelsitugramme, Fig. 2). Auch für die mathematische Behandlung der durch die Messungen ermittelten Werte dürften 30 Daten ein repräsentatives Minimum darstellen.

Für die situmetrischen Messungen an Steinstreifen wird die Meßtafel so orientiert, daß ihre Mittellinie (Radius zwischen den Sektoren 6 und 7 oder Winkelhalbierende des Poserschen Sektors I) in die jeweilige Fallinie weist, d. h. in die Richtung der Form. Die Tafelebene wird oberflächenparallel gelegt und paßt sich so der jeweiligen Hangneigung an (Furrer, 1965, 1966; Bachmann, 1966; Elsasser, 1966).

Auf Grund von 65 Messungen an rezenten Steinstreifen, vorgenommen nach der ursprünglichen Methode, d. h. ohne Aufteilung der steilgestellten Gerölle auf die Sektoren der Meßtafelebene, haben sich die bereits publizierten Ergebnisse herausgestellt (Furrer, 1966). Inzwischen sind 30 Messungen nach der verbesserten Methode dazugekommen. Alle Messungen sind an Formen in verschiedenen Gebieten ausgeführt worden, also in petrographisch verschiedenem Material, in verschiedenartiger Exposition und auf ungleichartig geneigten Hängen. Die in die Sektoren I, II, III und IV aufgeteilten Mittelwerte der vorliegenden Messungen präsentieren sich folgendermaßen:

$\begin{array}{lcccc}1 . & \text { I } & \text { II } & \text { III } & \text { IV } \\ 65 \text { rezente Stein- } & 58 \% & 142 / 3 \% & 211 / 3 \% & 6 \%\end{array}$
streifen, ursprüngliche Methode

2.

30 rezente Stein-

$\begin{array}{ccc}\text { I } & \text { II } & \text { III } \\ 541 / 2 \% & 201 / 2 \% & 25 \%\end{array}$
streifen, verbesserte Methode

Stellen wir diesen Mittelwerten, gewonnen an rezenten Formen, das aus 30 gleichermaßen vorgenommenen Messungen an fossilen Steinstreifen auf einer Riß-Schotter-Fläche im Ruckfeld ermittelte Ergebnis gegenüber:

3.

30 fossile Steinstreifen ursprüngliche Methode

$\begin{array}{cccc}\text { I } & \text { II } & \text { III } & \text { IV } \\ 62 \% & 7 \% & 28 \% & 3 \%\end{array}$

Aus diesen Meßergebnissen werden die Eigentümlichkeiten der Einregelung in Steinstreifen ersichtlich:

1. Die gestreckten Gerölle liegen so eingeregelt, daß ihre Längsachsen bevorzugt in die Richtung der Fallinie weisen. Sodann ist charakteristisch für Steinstreifen, daß auch quer zur Fallinie, also in der Richtung des Isohypsenverlaufs, wiederum ein höherer Wert festzustellen ist. Die in der Sammel- 


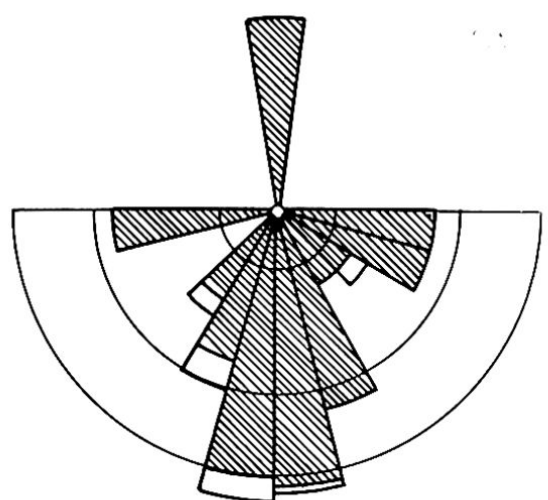

Steinstreifen

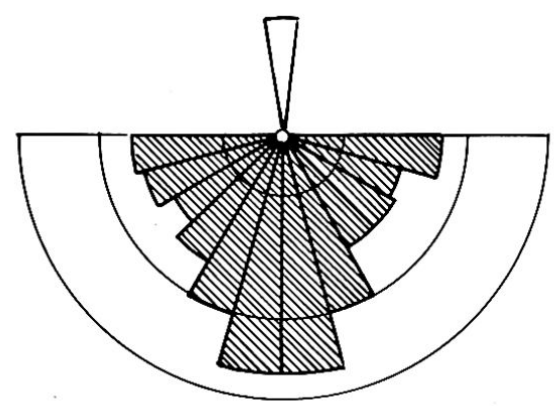

Steinstreifen

Einzelsitugramme

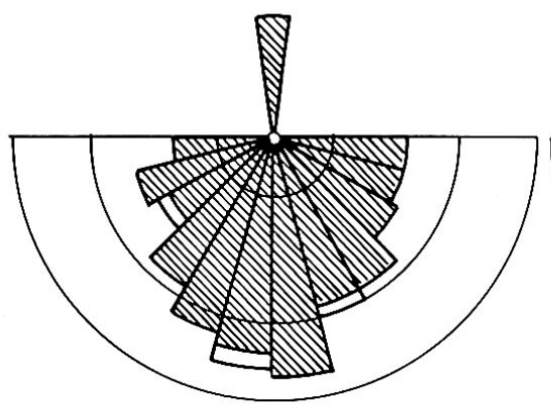

Zwischenstreifen

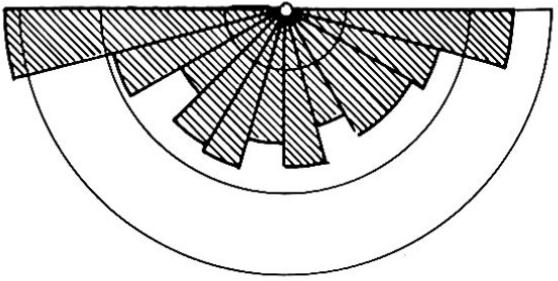

Flussschotter

Sammelsitugramme

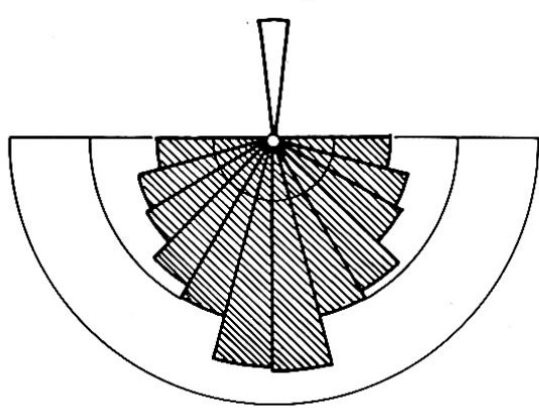

Zwischenstreifen

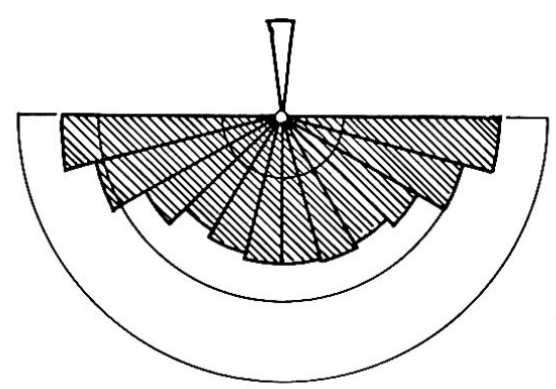

Flussschotter

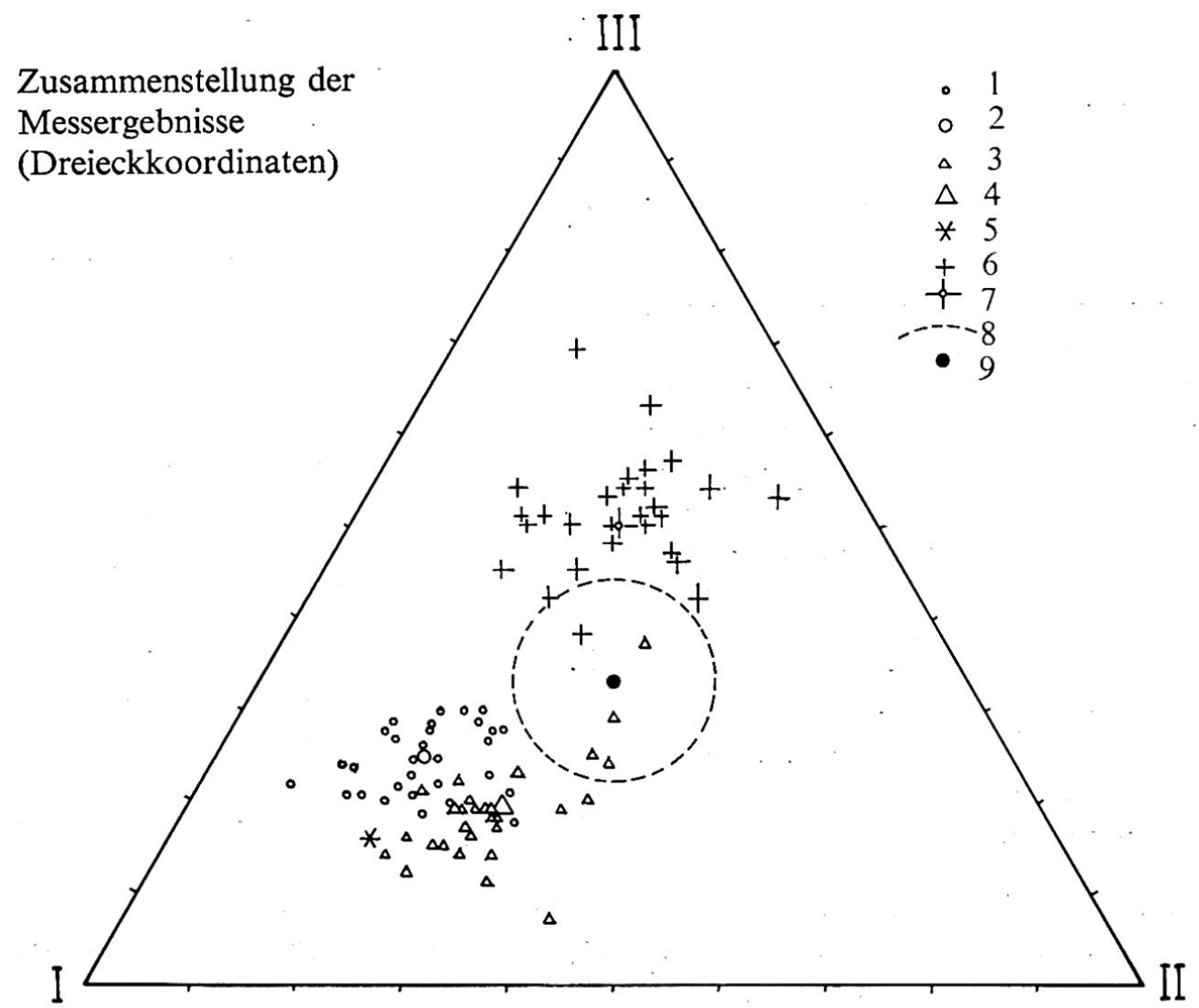


gruppe II auftretenden Werte fallen zurück, und die Anzahl der steilgestellten Gerölle nimmt sich recht bescheiden aus. Der Anteil der Längsachsen, die von der jeweiligen Fallinie nur bis zu höchstens $30^{\circ}$ abweichen, erscheint also am größten. Den zweiten Platz belegt der Anteil der Gerölle, deren Längsachsen mit der Fallinie Winkel von $60^{\circ}$ bis $90^{\circ}$ einschließen.

Diese entscheidende Hauptregel: I $>$ III $>$ II $>$ IV (Furrer, 1965) erwies sich bei den 65 Messungen nach ursprünglicher und den 30 Messungen nach verbesserter Methode an rezenten Formen in $88 \%$ bzw. $80 \%$ aller Fälle als erfüllt. Bei den 30 Messungen an fossilen Steinstreifen, vorgenommen nach der ursprünglichen Methode, ergaben $80 \%$ ein derartiges Ergebnis.

2. Im Mittel übersteigt der im Sektor I auftretende Wert $50 \%$. Es zeigt sich, daß bei den ersten 65 Messungen in $88 \%$, bei den 30 neuen Messungen an rezenten Formen in $77 \%$ und bei den Daten, die sich auf die fossilen Formen beziehen, gar in $97 \%$ aller Fälle der Anteil der Gerölle, deren Längsachsen in die Sammelgruppe I fallen, mehr als $50 \%$ ausmacht.

3. Alle Messungen sind mit der in 12 Sektoren zu je $15^{\circ}$ eingeteilten Meßtafel vorgenommen worden. Diese feinere Gliederung ließ die oben erwähnte Hauptregel noch prägnanter erscheinen. Es zeigte sich nämlich, daß von den in Sektor I fallenden Geröllelängsachsen mehr als die Hälfte beidseits nur um $0^{\circ}$ bis $15^{\circ}$ von der Fallinie abweichen. Somit wird die Summe der Sektoren 6 und 7 größer als die Summe der Sektoren 5 und 8. Diese Tatsache konnte bei den Messungen an rezenten Steinstreifen unter den ersten 65 Resultaten in 97\% der Fälle und bei den zweiten 30 Ergebnissen in jedem Einzelfall beobachtet werden. Auch bei den Messungen an fossilen Steinstreifen erwies sich diese Regel in jedem Fall als erfüllt.

4. Eine ähnliche Feststellung ist bei den Geröllelängsachsen möglich, die quer zur Fallinie eingeregelt liegen. Hier erfährt nämlich die Querstellung eine besondere Betonung dadurch, daß die Zahl der Geröllelängsachsen, die mit der Fallinie Winkel von $75^{\circ}$ bis $90^{\circ}$ einschließen, größer ist als die Zahl derjenigen, die nur um $60^{\circ}$ bis $75^{\circ}$ davon abweichen. Somit ist die Summe der Sektoren 1 und 12 größer als die Summe der Sektoren 2 und 11. Bei den rezenten Formen war dieser Umstand in bezug auf die ersten 65 Messungen in $94 \%$ und in bezug auf die zweiten 30 in $77 \%$ der Fälle feststellbar. Die an fossilen Formen gewonnenen Ergebnisse zeigen diese betonte Querstellung durchwegs.
Diese Regeln, die sich bei der Auswertung einer stets wachsenden Anzahl von Meßergebnissen nach und nach herausgeschält haben, kommen sowohl in den Situgrammen als auch bei der Darstellung im Dreieck deutlich zum Ausdruck (Fig. 2).

b) Die situmetrischen Verhältnisse in Zwischenstreifen

Anfänglich schienen die Messungen an rezenten Zwischenstreifen keine faßbaren Regeln erkennen zu lassen (Furrer, 1965). Wenn die Ergebnisse ins Dreieck übertragen werden, weisen "Zwischenstreifenwerte» eine bedeutend größere Streuung auf als "Streifenwerte» (Bachmann, 1966). Doch ordnen sich auch die Punkte, die situmetrischen Messungen an Zwischenstreifen entsprechen, einem bestimmten, allerdings weniger klar ersichtlichen Flächenstück ein. Die Scharung ist vor allem dadurch bedingt, daß sich bei situmetrischen Meßergebnissen von Zwischenstreifen ein mehr oder weniger betontes Maximum in der Sammelgruppe I einstellt. Damit schält sich die situmetrische Regel heraus, die für Solifluktionsmassen charakteristisch ist. Diese zeichnen sich nämlich dadurch aus, daß die von der Fallinie um $0^{\circ}$ bis höchstens $30^{\circ}$ abweichenden $\mathrm{Ge}$ röllelängsachsen den höchsten Prozentanteil aus: machen (Poser, 1951). Diesem Maximum in der Sammelgruppe I fügen sich bedeutend geringere Werte, die über die Sammelgruppen II, III und IV abfallen, bei. Die folgende Zusammenstellung beruht auf 28 Messungen, die an rezenten Formen nach der ursprünglichen Methode vorgenommen worden sind, und 30 Messungen, die auf der verbesserten Methode basieren. Entsprechend den Steinstreifen handelt es sich auch bei den Zwischenstreifen um Formen in verschiedenartigsten Verhältnissen.

\begin{tabular}{|c|c|c|c|c|}
\hline 1. & I & II & III & IV \\
\hline $\begin{array}{l}28 \text { rezente Zwischen- } \\
\text { streifen, ursprüngliche } \\
\text { Methode }\end{array}$ & $48 \%$ & $30 \%$ & $18 \%$ & $4 \%$ \\
\hline 2. & I & II & III & IV \\
\hline $\begin{array}{l}30 \text { rezente } Z \text { Zwischen- } \\
\text { streifen, verbesserte } \\
\text { Methode }\end{array}$ & $51 \%$ & $30 \%$ & $19 \%$ & $(4 \%)$ \\
\hline
\end{tabular}

Figur 2. Einzel- und Sammelsitugramme der in dieser Arbeit behandelten morphologischen Elemente sowie Zusammenfassung von Meßergebnissen, dargestellt mittels Dreieckkoordinaten.

Legende: 1 Einzelwerte von Steinstreifen; 2 «Schwerpunkt» (Mittelwert) der Steinstreifenwerte (vgl. Sammelsitugramm); 3 Einzelwerte von Zwischenstreifen; 4 «Schwerpunkt» der Zwischenstreifenwerte (vgl. Sammelsitugramm); 5 «Schwerpunkt» der Wanderblockwerte (vgl. Fig. 3); 6 Einzelwerte von Flußschottern; 7 "Schwerpunkt» der Schotterwerte; 8 Begrenzung des «Vertrauensbereiches» der Gleichverteilung; 9 Ideale Gleichverteilung (Mittelpunkt des Dreiecks).

Die Sammelsitugramme lassen erkennen, wie sich ein symmetrisches Bild einstellt, wenn genügend Ergebnisse vorliegen 


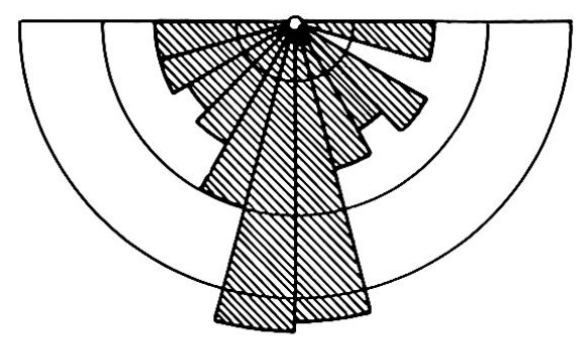

Figur 3. Das Sammelsitugramm der eingemessenen Wanderblöcke entspricht dem "Solifluktionssitugramm» in Zwischenstreifen

Die oben erwähnte Regel findet ihre Bestätigung ohne weiteres auch dann, wenn bei der verbesserten Methode die steilgestellten Gerölle in der $\mathrm{Zu}-$ sammenstellung nicht mehr in Erscheinung treten. Bei den ersten 28 Messungen stellte sich in $82 \%$ aller Fälle, bei den zweiten 30 sogar in $97 \%$ der Fälle ein Ergebnis ein, das der Regel I $>$ II $>$ III $>$ (IV) entspricht.

Interessant ist in diesem Zusammenhang das situmetrische Verhalten von Wanderblöcken. Unter 123 eingemessenen Beispielen weisen die Längsachsen von $65 \%$ der Exemplare in die Sammelgruppe I, $19 \%$ fallen in die Sammelgruppe II und 16\% sind der Sammelgruppe III zugehörig (Sammelsitugramm, Fig. 3). Es entsteht also ein Situgramm, das in seinem Aufbau dem Solifluktionssitugramm entspricht. Allerdings darf aus diesem Befund nicht a priori geschlossen werden, daß die Wanderblöcke eine Solifluktionserscheinung s. str. darstellen. Vielmehr ist zu beachten, daß, wie Schmid (1958) erwähnt, die Steine mit dem gefrierenden Boden senkrecht angehoben werden, dann aber beim Tauen in Richtung der Erdschwere absinken oder abgleiten und daß das langsame Frostwandern mit einer Drehbewegung der Steine im Sinne des geringsten Widerstandes verbunden ist. So spiegelt sich im Situgramm auf jeden Fall das so bedingte Einschwenken in die Fallinie wider.

\section{Flußschotter}

Während sich bei Steinstreifen und Zwischenstreifen situmetrische Regeln mit hoher Wahrscheinlichkeit manifestieren und darum in der Feldarbeit grundlegende Bedeutung erlangen, erscheinen die Schotter in dieser Beziehung noch recht problematisch.

Zingg (1935) weist darauf hin, wie flache und flachstenglige Gerölle das Bestreben haben, sich quer zur Strömungsrichtung zu betten. Richter (1936) stellt bei situmetrischen Messungen vor norwegischen Gletschern fest, daß relativ große Gerölle sich quer zur Strömungsrichtung einstellen, während kleinere mit ihren Längsachsen eher in die Flu $\beta$ richtung weisen. Dies läßt den Schluß zu, daß die größeren Geschiebekomponenten, die beim Transport rollend fortbewegt werden, senkrecht zur Transportrichtung zur Ruhe kommen, während die kleineren flottieren und sich daher in die Fließrichtung einregeln. Allerdings spielt auch die Geschwindigkeit der Strömung eine Rolle und modifiziert die situmetrischen Verhältnisse. Poser und Hövermann (1951) gelangen ihrerseits zum Ergebnis, daß in Flußschottern die Längsachsen der gestreckten Gerölle sich vorwiegend quer zur Transportrichtung einstellen. Das Mittel der acht im Harz gewonnenen Meßergebnisse lautet folgendermaßen:

1.

8 Messungen

$\begin{array}{cccc}\text { I } & \text { II } & \text { III } & \text { IV } \\ 17 \% & 26 \% & 48 \% & 9 \%\end{array}$

Methode Poser und Hövermann (1951)

Somit gelangt hier die Querstellung recht klar zum Ausdruck. In der Strömungsrichtung hingegen tritt kein hervorstechender Wert auf. Demgegenüber machte aber Portmann (1954) die Beobachtung, daß die Mehrzahl der Gerölle in die Strömungsrichtung gedreht worden ist. Die Tatsache, daß die Einregelung bei Flußschottern vorwiegend quer zur Strömung erfolgt, hält ebenfalls Köster (1964) fest. Der Anteil der Sammelgruppe III schwankt zwischen 40 und $60 \%$. Doch entgeht auch diesem Autor nicht, daß erhebliche Abweichungen von dieser Regel eintreten können. Er sucht die Gründe dafür einerseits beim Transportmedium selbst (wechseln. de Stromstärken bei Hochwasser, scheinbare Nicht. einregelung auf Verwilderungssohlen), anderseits in der Beschaffenheit des Untergrundes (Uneben. heiten und unterliegende Schotterpackungen, wel. che die "Normaleinregelung» beeinflussen), unc schließlich in der Form der Gerölle. Partikel, die in Verhältnis von Länge, Breite und Höhe nur gering $\epsilon$ Differenzen aufweisen, sollen für widersinnige Ein regelung geradezu prädestiniert sein.

Wir fügen den bereits vorgenommenen Messunger 30 neue Ergebnisse bei. Gewonnen wurden sie teil. weise an Aufschlüssen und teilweise in der rezenter Schotterfüllung von Fluß- und Bachbetten. Füll. material und ausnehmend große Komponenter schieden bei der situmetrischen Untersuchung aus Nur Gerölle mit ausgesprochen deutlich erkenn barer Längsachse gelangten zur Einmessung.

Das gemittelte Ergebnis ist in folgender Ubersich zusammengefaßt:

2. 30 Messungen $\quad 24 \frac{1}{2} \% \quad 25 \frac{1}{2} \% \quad 50 \% \quad(21 / 3 \%$ verbesserte Methode

Im Vergleich zu den bisher publizierten Beobach tungen dürfen wir festhalten, daß unsere Ergebniss insofern mit denjenigen von Poser und Hövermant übereinstimmen, als die quer zur Strömung einge regelten Gerölle den maximalen Anteil ausmachen Der Prozentwert liegt innerhalb der von Köster er 


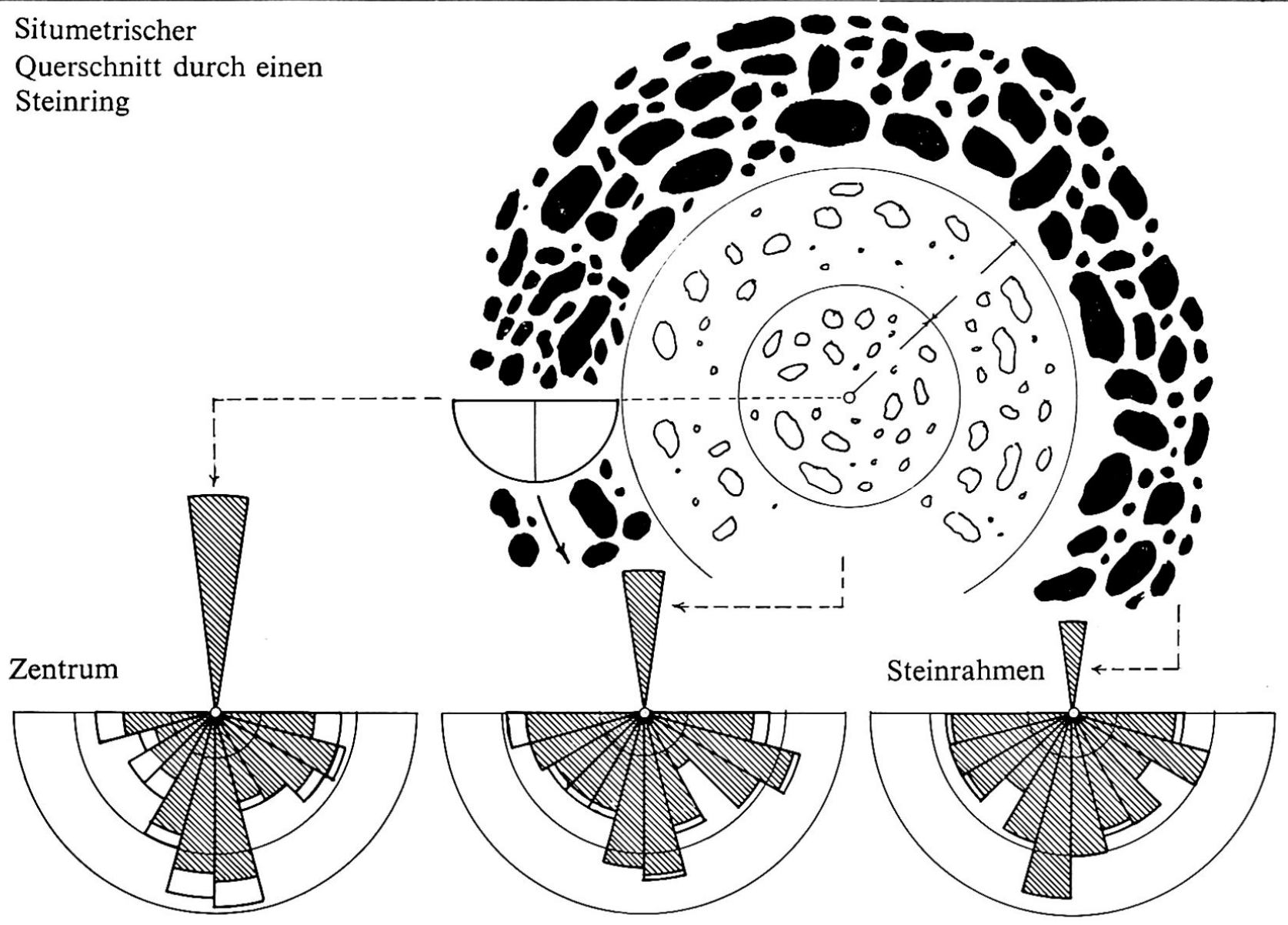

Figur 4. Die Situgramme lassen erkennen, wie in einem Steinring der Anteil der steilgestellten Partikel vom Zentrum gegen den Steinrahmen hin abnimmt, aber auch, daß sich bereits im Zentrum die für Strukturbodenformen bezeichnende Einregelung eingestellt hat

wähnten Spanne. Hingegen tritt in der Strömungsrichtung (Sammelgruppe I) weder ein Maximum in Erscheinung, wie es Portmann feststellte, noch aber das Minimum, wie es die Messungen von Poser und Hövermann ausdrücken. Allerdings darf vielleicht gesagt werden, daß 8 Messungen allein noch kein repräsentatives Mittel gewährleisten. Es ist darum durchaus möglich, daß sich bei einer größeren Anzahl von Messungen Mittelwerte eingestellt hätten, die den unsrigen entsprächen.

Das sich bei den 30 Messungen, von einem einzigen Fall abgesehen, durchwegs einstellende Maximum in der Sammelgruppe III bewirkt, daß sich bei der Darstellung in Dreieckkoordinaten die "Schotterpunkte» einem bestimmten Feld in der Dreiecksfläche einordnen (Fig. 2). Einer allein fällt ins durch Berechnung ermittelte "Gleichverteilungsfeld». Beachtenswert erscheint jedoch die eindeutige Scheidung der "Strukturbodenpunkte» von den "Schotterpunkten». In keiner Weise kommt es je zur Überlagerung. So bestätigt die graphische Darstellung die eingangs erwähnte Feststellung, daß es anhand der situmetrischen Befunde möglich ist, Strukturbodenformen von Schottern zu unterscheiden.

Das Sammelsitugramm in Figur 2 läßt erkennen, was schon in der zahlenmäßigen Zusammenstellung zum Ausdruck kommt, nämlich daß sich in den Sammelgruppen I, II und III die Mittelwerte verhalten wie $1: 1: 2$. Auch im ausgewählten Einzelsitugramm schimmert dieses Verhältnis durch. Allerdings ist bei den einzelnen Meßwerten die Streuung sehr beträchtlich, bedeutend stärker nämlich als bei den Ergebnissen von Strukturbodenformen.

Eine endgültige Abklärung des hier aufgeworfenen Problems ist vorderhand noch nicht möglich, denn es bedarf dazu noch einer bedeutend größeren Zahl von Einzelbeobachtungen.

\section{Einsatz der}

Situmetrie zur Bearbeitung von Detailproblemen

Die Situmetrie erlaubt die quantitative Erfassung von Formmerkmalen und liefert somit von subjek- 
tiven Einflüssen weitgehend unabhängige Resultate, die einen hohen Aussagewert erlangen. Im vorangehenden Kapitel ist dargelegt worden, wie der situmetrische Befund eine sichere Diagnose ermöglicht, wenn die erwähnten Grundregeln feststellbar sind. Darüber hinaus aber vermag die Situmetrie bei tiefer greifenden Untersuchungen neue Perspektiven zu eröffnen und den Weg zum besseren Verständnis der Formen und ihrer Entstehung zu ebnen.

Wir möchten dies an Beobachtungen in Steinringen und Steinstreifen erläutern.

\section{Situmetrischer Querschnitt durch einen Steinring}

Steinstreifen und Steinringe sind verwandte Formen. Dieselben Kräfte haben sie geschaffen, und durch die Situmetrie läßt sich die genetische Gleichartigkeit erfassen. Die gleichen Regeln manifestieren sich:

Die Steinstreifen passen sich in ihrem Verlaufe dem Verlauf der jeweiligen Fallinie an. Innerhalb der Form liegen die Längsachsen der gestreckten Gerölle vorwiegend in diese Richtung eingeregelt. Eine zweite Hauptrichtung, die sich wiederum durch einen verhältnismäßig hohen prozentualen Anteil auszeichnet, ist durch die Isohypsen gegeben. Beim Steinkreis hingegen liegen im Rahmen die meisten der gestreckten Gerölle so, daß ihre Längsachsen die gleiche Richtung einnehmen wie die Tangente an die geschlossene Form, während ein weiterer hervorstechender Anteil der den Rahmen bildenden Steine mit der Längsachse gegen das Zentrum hinweist. Der Richtung der Fallinie beim Steinstreifen entspricht also beim Steinring die Richtung der Tangenten, und die isohypsentreue Anordnung findet in der radialen Einregelung ihr Pendant. Auf Grund dieser Erkenntnis ergab sich, auf welche Weise situmetrische Messungen an Steinringen durchzuführen sind: Die Meßtafel wird so der geschlossenen Form entlang geführt, daß ihre Mittellinie stets die Rich- tung der Tangente einnimmt. So fangen die Sektoren 5 bis 8 , also die Sammelgruppe I, die tangential eingeregelten Gerölle ein, und in der Sammelgruppe III, also in den Sektorensummen $(1+2)+(11+$ 12), kommt die radial gerichtete Einregelung zum Ausdruck.

Wie erwähnt, überwiegen also bei Steinringen die tangential gelagerten Gerölle, die in der Regel einen Anteil von über $50 \%$ ausmachen, wobei die der Mittellinie unmittelbar benachbarten Sektoren 6 und 7 den höheren Wert aufweisen als die weiter abstehenden Sektoren 5 und 8. In bezug auf die radiale Richtung ist in der Regel die Summe der Sektoren 1 und 12 größer als diejenige der Sektoren 2 und 11. Die an den Steinstreifen erkannte Grundregel: I $>$ II $>$ III $>$ (IV) findet sich also wieder, ebenso die andern dort erwähnten Regeln.

Wie verändern sich aber innerhalb eines Steinringes die situmetrischen Verhältnisse? Die Untersuchung eines fossilen Ringes nach der ursprünglichen Methode ergab folgendes Ergebnis:

Innerhalb eines eng gehaltenene Kreises um den Ringmittelpunkt erreichten die steilgestellten Komponenten (IV) einen ausgesprochen hohen Wert, während sich in der Tafelebene keine interpretierbare Anordnung erkennen ließ. Im anschließenden Kreisring, begrenzt durch die Peripherie des Zentralkreises und den Innenrand des Steinrahmens, erfuhr der Wert der aufgerichteten Komponenten eine wesentliche Verringerung. Das für Steinringe typische Situgramm aber prägte sich erst bei der dritten Messung aus, die im Steinrahmen vorgenommen wurde (Bachmann, 1966).

Das in Figur 4 dargestellte Meßergebnis entspricht einer gleich angelegten Untersuchung an einem Steinring in Serpentingestein auf dem Weißfluhjoch (Boesch, 1967), die aber nach unserer verbesserten Methode vorgenommen worden war. Die der Darstellung zu Grunde liegenden Daten seien vollständig wiedergegeben:

\section{Zentraler Kreis}

$\begin{array}{lrrrrrrrrrrrrr}\text { Sektoren } & 1 & 2 & 3 & 4 & 5 & 6 & 7 & 8 & 9 & 10 & 11 & 12 & \\ \text { Werte } & 7 & 3 & 5 & 5 & 10 & 17 & 18 & 5 & 5 & 8 & 9 & 8 & \\ \begin{array}{l}\text { Davon } \\ \text { steilgestellt }\end{array} & 3 & 1 & 0 & 2 & 2 & 4 & 4 & 1 & 0 & 2 & 1 & 3 & \\ \text { kantengestellt } & 2 & 0 & 1 & 0 & 1 & 0 & 1 & 0 & 0 & 0 & 0 & 1 & \\ \end{array}$

\section{Kreisring}

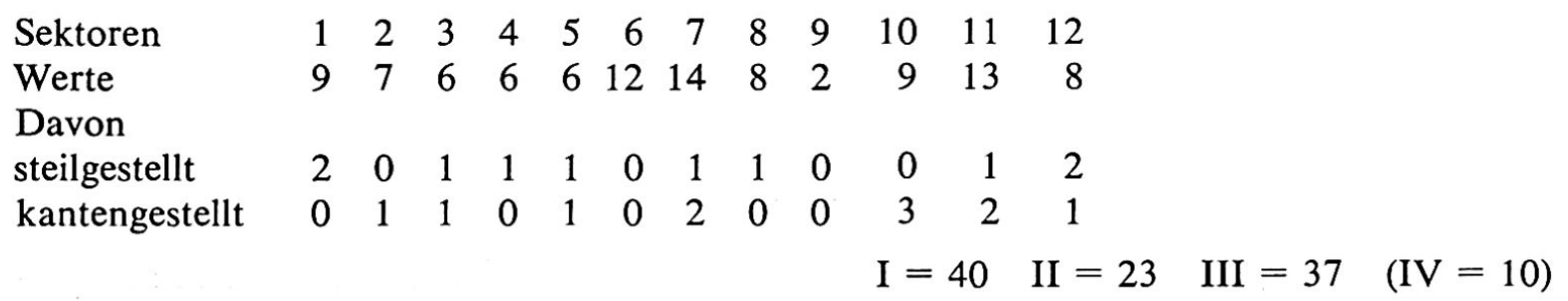




\section{Steinrahmen}

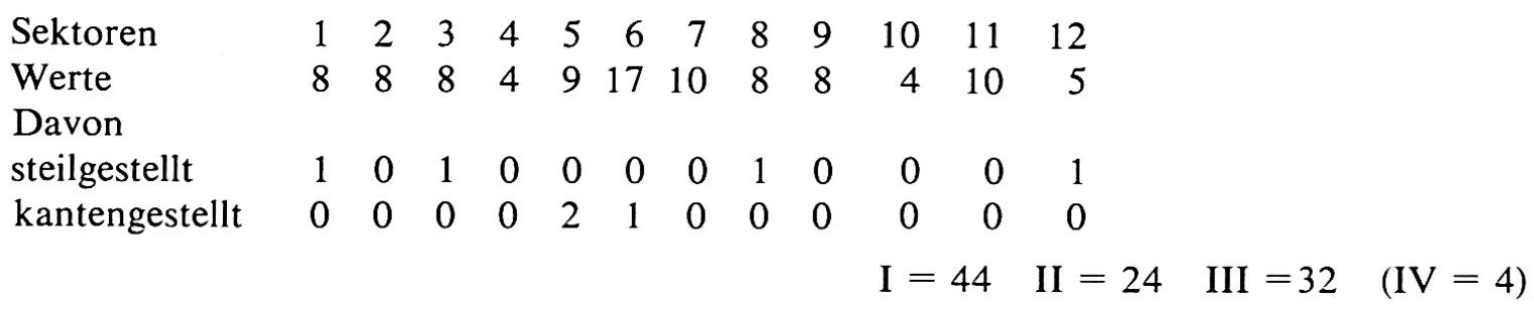

Aus diesen Beobachtungen geht hervor, daß im Zentrum eines Steinkreises der Anteil der steilgestellten Komponenten verhältnismäßig hoch ist und sich dann gegen den Steinrahmen hin vermindert. Sehr wahrscheinlich hängt diese Tatsache mit dem Ausfrieren der Steine zusammen, das vorwiegend im Zentrum der Feinerdesäule erfolgt. Die nach der verbesserten Methode vorgenommene Messung erzielt aber ein sehr auffallendes Ergebnis. Nicht nur die bereits gewonnene Erkenntnis erfährt ihre Bestätigung, sondern auch eine weitere Besonderheit wird offenbar. Projiziert man die Richtung der Längsachsen von steilgestellten Geröllen auf die Tafelebene, so erhellt, daß sich die für Strukturbodenformen als typisch erkannte Einregelung bereits schon im Zentrum des Ringes eingestellt hat. Alle 3 Messungen weisen das Maximum in der Sammelgruppe I, den nächsttieferen Wert aber in der Sammelgruppe III auf, während die Sammelgruppe II zurücktritt. Somit äußert sich das spezifische Ordnungsprinzip bereits dann, wenn die Form als solche noch gar nicht ausgebildet erscheint. Für die

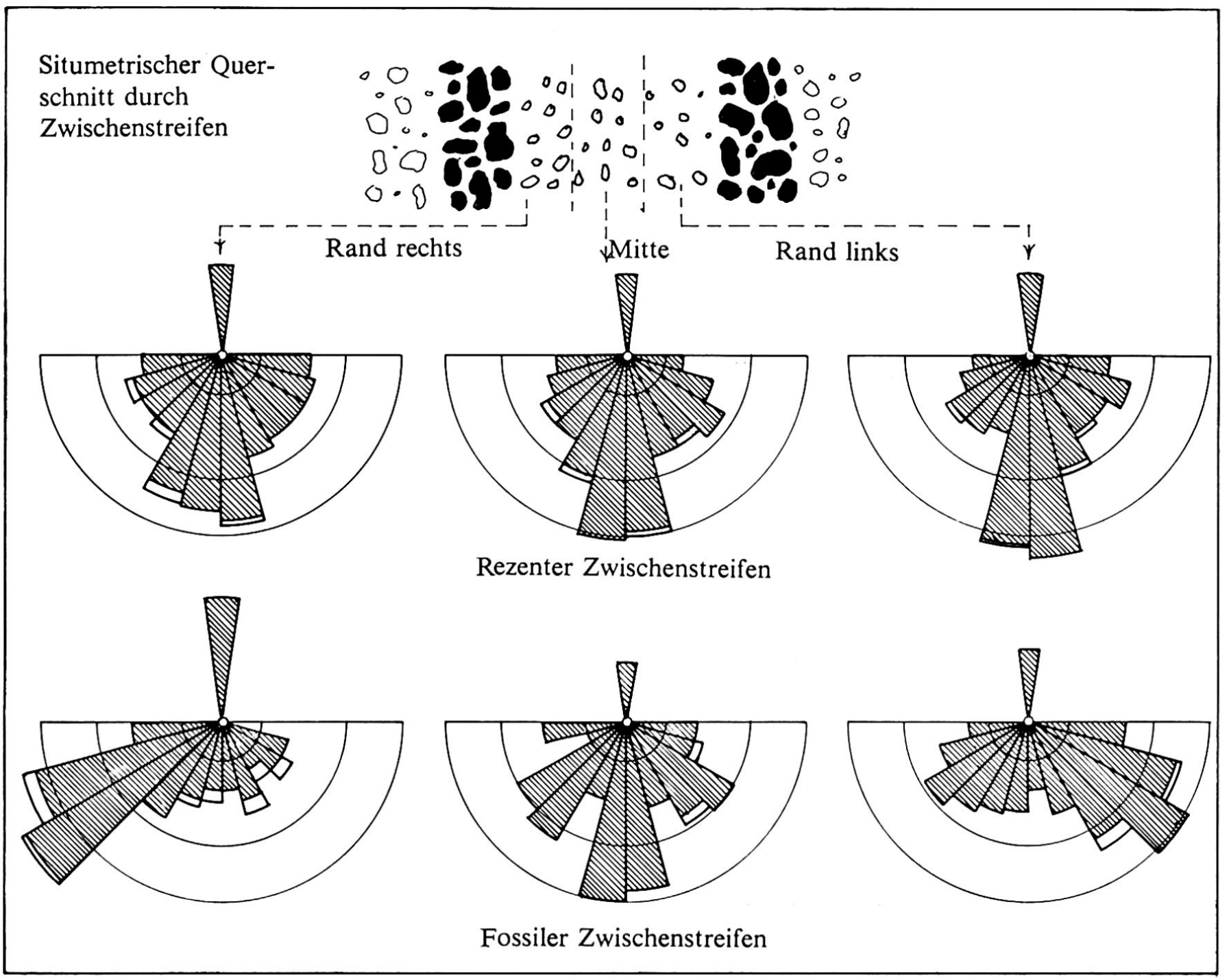

Figur 5. Die Situgramme veranschaulichen, wie die Fließrichtung in der Mitte des Zwischenstreifens der Fallinie entspricht, an den Rändern aber schräg hangabwärts gegen die Steinrahmen zielt 
Feldarbeit ist dies von höchster Bedeutung, weil dadurch eine Möglichkeit geboten wird, bestimmte Formen auch bei oberflächlich kaum erkennbarer Ausbildung - z. B. geringer Reifegrad bei rezenten oder hoher Zerstörungsgrad bei fossilen Bildungen - einwandfrei als solche zu identifzieren. In dieser Hinsicht ist also unsere verbesserte situmetrische Methode der ursprünglichen überlegen.

\section{Situmetrischer Querschnitt durch einen Zwi- schenstreifen}

Die allgemeine situmetrische Behandlung von Zwischenstreifen ergab, daß die Meßwerte in hohem Maße um ihren Mittelwert streuen und die sich her- ausstellende Regel I > II > III nicht mit derselben Deutlichkeit erfüllt wurde wie die entsprechend $\epsilon$ Regel bei den Steinstreifen, nämlich I $>$ III $>$ I] (Fig. 2). Wir zögern daher, jetzt schon weittragend $\epsilon$ Schlußfolgerungen zu ziehen. Doch könnte die fol. gendermaßen angelegte Einzeluntersuchung einer Fingerzeig geben:

Bei je einem rezenten (Fuorcla da Fàller) und einer fossilen Zwischenstreifen (Ruckfeld) wurde eint erste Messung in seiner Mittellinie, also gewisserma Ben im Stromstrich, vorgenommen und hierauf j eine weitere links und rechts davon. Der Zwischen streifen wurde somit in einen Mittel- und 2 Rand streifen unterteilt. Dabei stellten sich die anschlie Bend aufgezeichneten und in Figur 5 verarbeiteter Ergebnisse ein:

1. Rezenter Zwischenstreifen (Fuorcla da Fàller, Elsasser, 1966)

a) Mittelstreifen

\begin{tabular}{|c|c|c|c|c|c|c|c|c|c|c|c|c|}
\hline Sektoren & 1 & 2 & 3 & 4 & 5 & 6 & 7 & 8 & 9 & 10 & 11 & 12 \\
\hline Werte & 3 & 4 & 6 & 6 & 11 & 21 & 20 & 7 & 7 & 8 & 5 & 2 \\
\hline $\begin{array}{l}\text { Davon } \\
\text { steilgestellt }\end{array}$ & 0 & 0 & 1 & 0 & 1 & 0 & 1 & 0 & 1 & 0 & 0 & 0 \\
\hline
\end{tabular}

b) Randstreifen rechts (Blickrichtung hangabwärts)

$\begin{array}{lrrrrrrrrrrrrr}\text { Sektoren } & 1 & 2 & 3 & 4 & 5 & 6 & 7 & 8 & 9 & 10 & 11 & 12 & \\ \begin{array}{l}\text { Werte } \\ \begin{array}{l}\text { Davon } \\ \text { steilgestellt }\end{array}\end{array} & 4 & 6 & 5 & 6 & 15 & 16 & 18 & 7 & 6 & 6 & 6 & 5 & \\ & 0 & 1 & 0 & 1 & 2 & 0 & 1 & 0 & 0 & 0 & 0 & 0 & \\ & & & & & & & & & & \text { I = 56 } & \text { II = 23 } & \text { III }=21\end{array}$

c) Randstreifen links

$\begin{array}{lrrrrrrrrrrrrr}\text { Sektoren } & 1 & 2 & 3 & 4 & 5 & 6 & 7 & 8 & 9 & 10 & 11 & 12 & \\ \begin{array}{l}\text { Werte } \\ \begin{array}{l}\text { Davon } \\ \text { steilgestellt }\end{array}\end{array} & 2 & 3 & 6 & 5 & 4 & 23 & 25 & 10 & 6 & 5 & 7 & 4 & \\ & 0 & 0 & 1 & 0 & 0 & 1 & 0 & 1 & 1 & 0 & 0 & 0 & \\ & & & & & & & & & & \text { I }=62 & \text { II }=22 & \text { III }=16\end{array}$

2. Fossiler Zwischenstreifen (Ruckfeld, Bachmann, 1967)

a) Mittelstreifen

$\begin{array}{lrrrrrrrrrrrrr}\text { Sektoren } & 1 & 2 & 3 & 4 & 5 & 6 & 7 & 8 & 9 & 10 & 11 & 12 & \\ \begin{array}{l}\text { Werte } \\ \begin{array}{l}\text { Davon } \\ \text { steilgestellt }\end{array}\end{array} & 4 & 1 & 10 & 12 & 4 & 20 & 18 & 5 & 9 & 10 & 4 & 3 & \\ & 0 & 0 & 0 & 0 & 0 & 0 & 0 & 0 & 1 & 0 & 1 & 0 & \\ & & & & & & & & & & \mathrm{I}=47 & \mathrm{II}=41 & \text { III }=12\end{array}$


b) Randstreifen rechts

$\begin{array}{lrrrrrrrrrrrr}\text { Sektoren } & 1 & 2 & 3 & 4 & 5 & 6 & 7 & 8 & 9 & 10 & 11 & 12 \\ \begin{array}{l}\text { Werte } \\ \begin{array}{l}\text { Davon } \\ \text { steilgestellt }\end{array}\end{array} & 5 & 26 & 33 & 8 & 5 & 4 & 3 & 6 & 3 & 4 & 3 & 0 \\ & 0 & 3 & 1 & 0 & 1 & 1 & 0 & 2 & 1 & 1 & 0 & 0\end{array}$

c) Randstreifen links

\begin{tabular}{|c|c|c|c|c|c|c|c|c|c|c|c|c|}
\hline Sektoren & 1 & 2 & 3 & 4 & 5 & 6 & 7 & 8 & 9 & 10 & 11 & 12 \\
\hline Werte & 2 & 5 & 9 & 7 & 6 & 5 & 3 & 6 & 14 & 21 & 16 & 6 \\
\hline $\begin{array}{l}\text { Davon } \\
\text { steilgestellt }\end{array}$ & 0 & 0 & 0 & 0 & 0 & 0 & 0 & 0 & 2 & 0 & 1 & 0 \\
\hline
\end{tabular}

Beachtenswert ist, wie in beiden Fällen im Stromstrich des Zwischenstreifens ein mehr oder weniger symmetrisches Situgramm auftritt. Einwandfrei hebt sich der höchste Wert in der Sammelgruppe I vom kleineren in der Gruppe II und dem noch kleineren in der Sammelgruppe III ab. Bei der rezenten Form stellt sich auch in den randlichen Zwischenstreifenpartien das Solifluktionssitugramm ein, jedoch mit einer offensichtlichen Modifikation, die in der $\mathrm{Zu}$ sammenfassung in die Sammelgruppen I, II und III nicht zum Ausdruck kommt: In der Lageverteilung der Längsachsen gibt sich eine bestimmte Tendenz zu erkennen. Die gestreckten Gerölle haben das Bestreben, in der Fließrichtung hangabwärts gegen den Rand hin, d. h. gegen die den Zwischenstreifen begrenzenden Steinstreifen auszubrechen. So erscheint, besonders klar erkennbar bei der fossilen Form, am rechten Zwischenstreifenrand die rechte und am linken die linke Hälfte des Situgramms erheblich stärker belastet. Auf der rechten Seite des rezenten Zwischenstreifens weichen 52\% der eingemessenen Längsachsen nach rechts von der Fallinie ab und auf der entsprechenden Seite der fossilen Form gar $81 \%$. Auf der linken Seite sind es beim rezenten $\mathrm{Zwischenstreifen} 57 \%$ und beim fossilen $66 \%$ der Längsachsen, die nach links weisen. Liegt hier vielleicht eine Erklärung für die erhebliche Streuung der an Zwischenstreifen gewonnenen Meßergebnisse vor? Jedenfalls wird die für die Ermittlung der Dreieckkoordinaten nötige Zusammenfassung der 12 Einzelergebnisse in 3 Werten dem tatsächlichen Befund nicht mehr gerecht. Hingegen leistet das Situgramm bessere Dienste, weil es die spezifischen Modifikationen genau widerspiegelt und Hinweise auf den Fließcharakter in Zwischenstreifen zu geben vermag.

Auch Kommissuren, die sich in spitzen Winkeln von einem Steinstreifen abspalten und den Zwischenstreifen schief queren, um sich dann wiederum in spitzen Winkeln mit dem benachbarten Streifen zu vereinigen, beeinflussen die situmetrischen Meßergebnisse. Doch weisen derartige Bildungen die gleichen Merkmale auf wie die Steinstreifen selbst, nämlich ein Maximum in der "Fließrichtung» und den nächsttieferen Wert quer dazu. Durch die situmetrische Untersuchung läßt sich also eine Kommissur lokalisieren. Zudem ist es möglich, an Hand der Ergebnisse ihre Richtung exakt zu bestimmen, auch wenn die Form als solche gar nicht sichtbar ist. Dies ist wiederum ein besonderer Vorteil bei der Bearbeitung von fossilen Strukturbodenformen (Bachmann, 1966).

\section{Zusammenfassung}

Die Einregelung, d. h. die Lage der Längsachsen der Einzelgerölle, ist ein wesentliches inneres Strukturmerkmal von Formen und Ablagerungen. Die Situmetrie, welche die quantitative Erfassung dieser Einregelung zum Ziele hat, ist an sich zwar keine neue Methode, doch ist es gelungen, sie durch verfeinertes Vorgehen $\mathrm{zu}$ einem fundamentalen morphologischen Untersuchungs-Hilfsmittel $z z$ entwickeln. Ebenso wurde es möglich, durch adäquate graphische Darstellungsweisen, nämlich durch das Situgramm und durch die Dreieckkoordinaten, die Aussagekraft der Meßergebnisse zu erhöhen. Die Dreieckkoordinaten erlauben zudem die mathematische Behandlung einer Vielzahl von Ergebnissen. Es ist möglich, mit hoher Wahrscheinlichkeit eine Gleichverteilung auszuschließen und die Situmetrie bei schlecht oder überhaupt nicht erkennbaren Formen als diagnostisches Hilfsmittel einzusetzen. Dies wird am Beispiel von Solifluktionsformen, insbesondere von Steinstreifen und Zwischenstreifen sowie an Flußschottern erläutert. Sowohl den Strukturbodenformen als auch den Flußschottern liegen bestimmte Einregelungsprinzipien zu Grunde, die sich 
als situmetrische Regelmäßigkeiten äußern. Diese Regelmäßigkeiten sind bei Strukturbodenformen klar erkennbar, während die Flußschotter in dieser Beziehung vorderhand noch problematisch erscheinen. Doch nicht nur als diagnostisches Hilfsmittel, sondern auch für die Analyse von Einzelformen läßt sich die Situmetrie in der verfeinerten Art zweckmäßig einsetzen. Besondere Bedeutung kommt ihr im Hinblick auf die Untersuchung fossiler Formen zu.

\section{Literatur}

Bachmann, F.: Fossile Strukturböden und Eiskeile auf jungpleistozänen Schotterflächen im nordostschweizerischen Mittelland. Zürich 1966.

Boesch, M.: Beobachtungen an subnivalen Bodenformen im Gebiet Weißfluhjoch/Davos GR. Dipl. Arb., Zürich 1967.

Elsasser, H.: Untersuchungen an Erdbülten und Strukturböden im Avers. Dipl. Arb., Zürich 1966.

Fischer, K.: Zur Anwendung der morphometrischen Schotteranalyse bei Untersuchungen in Alpentälern. Zeitschrift für Geomorphologie, Heft 1, 1966. Furrer, G.: Die Höhenlage von subnivalen Bodenformen. Zürich 1965.
Furrer, G.: Die subnivale Höhenstufe und ihre Un. tergrenze in den Bündner und Walliser Alpen. Geo. graphica Helvetica, 4, 1965.

Furrer, G.: Beobachtungen an rezenten und fossilen Strukturböden. Experientia 22, 489, 1966.

Körber, H.: Morphologie von Waldeck und Ostsauerland. Würzburger geogr. Arb. Würzburg 1956. Köster, E.: Granulometrische und morphometrische Meßmethoden. Stuttgart 1964.

Portmann: Pétrographie des moraines du glacier würmien du Rhône dans la région des lacs subjurassiens. Bull. Soc. neuchâteloise de géogr. t. 51, fasc. $5,1954 / 55$.

Poser, H. und Hövermann, J.: Untersuchungen zur pleistozänen Harzvergletscherung, Abh. Braunschw. Wiss. Ges. III, 1951.

Richter, K.: Die Bewegungsrichtung des Inlandeises, rekonstruiert aus den Kritzen und Längsachsen der Geschiebe. Zeitschrift für Geschiebeforschung: Bd. 8, Heft 1, 1932.

Richter, K.: Gefügestudien im Engebrae, Frondalsbrae und ihren Vorlandsedimenten. Zeitschrift für Gletscherkunde, Bd. 24, 1936.

Rydquist, F.: Studier inom Öländska Polygonmarker. Stockholm 1960.

Schmid, J.: Rezente und fossile Frosterscheinungen im Bereich der Gletscherlandschaft der Guglei Ache (Ötztaler Alpen). Schlern Schriften 190, 1958.

Zingg, T.: Beitrag zur Schotteranalyse. Zürich 1935. 